\title{
Understanding amyloid aggregation by statistical analysis of atomic force microscopy images
}

\author{
Jozef Adamcik', Jin-Mi Jung², Jérôme Flakowski ${ }^{3}$, Paolo De Los Rios ${ }^{3}$, Giovanni Dietler ${ }^{1 \star}$ and \\ Raffaele Mezzenga4*
}

The aggregation of proteins is central to many aspects of daily life, including food processing, blood coagulation, eye cataract formation disease and prion-related neurodegenerative infections $^{1-5}$. However, the physical mechanisms responsible for amyloidosis-the irreversible fibril formation of various proteins that is linked to disorders such as Alzheimer's, Creutzfeldt-Jakob and Huntington's diseases-have not yet been fully elucidated ${ }^{6-9}$. Here, we show that different stages of amyloid aggregation can be examined by performing a statistical polymer physics analysis of single-molecule atomic force microscopy images of heat-denatured $\beta$-lactoglobulin fibrils. The atomic force microscopy analysis, supported by theoretical arguments, reveals that the fibrils have a multistranded helical shape with twisted ribbon-like structures. Our results also indicate a possible general model for amyloid fibril assembly and illustrate the potential of this approach for investigating fibrillar systems.

Protein self-assembly is a wide-ranging phenomenon and is of great importance in several areas of science. The reversible formation of fibrils from globular proteins is a phenomenon occurring naturally, in vivo, for proteins such as actin and tubulin ${ }^{10,11}$. Other well-known examples of protein fibrillation include the irreversible amyloid fibril formation of various proteins implicated in neurological disorders such as Alzheimer's, Creutzfeldt-Jakob or Huntington's diseases. Typically, these fibrils have long, unbranched, and often twisted structures that are a few nanometres in diameter ${ }^{1,8}$. However, many peptides and proteins, including many globular food proteins that are used as gelling agents, foaming agents or emulsifiers, can also form amyloid-like structures in vitro. Such structures can possess desirable mechanical properties and can be used to create useful textures and structures ${ }^{12,13}$.

An example of a fibril formation of globular proteins is provided by the fine-stranded heat-set gels formed by heating solutions of various globular food proteins such as ovalbumin, bovine serum albumin $^{12}$ and $\beta$-lactoglobulin ${ }^{13}$. $\beta$-Lactoglobulin has been particularly well studied, because it represents both a relevant model system and a major whey protein of interest to the food industry ${ }^{14-22}$.

Despite the fact that the individual parameters controlling the aggregation of globular proteins into amyloid fibrils have been well identified, the driving force for such an aggregation process still remains obscure. Studies devoted to the characterization of fibril structures based on light, neutrons and X-ray scattering methods, being bulk techniques, have only provided an average ensemble picture of the fibrils ${ }^{23}$. Single-molecule techniques such as atomic force microscopy (AFM) and transmission electron microscopy (TEM) have recently emerged to probe amyloid fibrils at the molecular level ${ }^{24-27}$. Nevertheless, so far, a fully comprehensive picture of the aggregation behaviour among different amyloid fibrils has not yet been achieved. In the present work we have used single-molecule AFM to provide molecular details regarding individual $\beta$-lactoglobulin amyloid fibrils and fibril aggregates obtained by heat denaturation at $\mathrm{pH} 2$. We have then combined the statistical theory of semi-flexible polymers with simple scaling arguments to determine their hierarchical structures and aggregation behaviour at the same $\mathrm{pH}$ conditions $(\mathrm{pH} 2)$, and thus in the regime of high linear charge densities.

The AFM images of $\beta$-lactoglobulin fibrils produced by heatdenaturation at $\mathrm{pH} 2$ presented in Fig. 1a-d show several micrometre-long semiflexible fibrils, in agreement with previous literature reports ${ }^{18,21}$. Because width determination is strongly dependent on the size and shape of the AFM tip, maximum height measurements were preferred to characterize the different fibril cross-sections. By performing a statistical analysis on AFM images we were able to determine precisely the contour length (Fig. 1e) and cross-section (Fig. 1f) of $\beta$-lactoglobulin fibrils. The most crowded fibril population has a maximum height centred at $4.0 \pm 0.5 \mathrm{~nm}$. Features from this population agree well with other AFM studies on $\beta$-lactoglobulin fibrils obtained after heating at $80{ }^{\circ} \mathrm{C}$ and pH 2 (refs 17,25). Figure $1 \mathrm{f}$ shows that some very fine filaments of height $2.0 \pm 0.4 \mathrm{~nm}$ can also be observed, together with thick fibrils of height $6.0 \pm$ $0.4 \mathrm{~nm}$. Surprisingly, Fig. If also shows that very thick fibrils of heights well beyond $6 \mathrm{~nm}$ are also occasionally observed. The physical meaning of these thick fibrils will be discussed later.

Other valuable structural details, such as fibril splitting (Fig. 2a) or fibril thinning (Fig. 2b), can be resolved by AFM measurements of individual fibrils. In both cases, the height of the thicker parts was found to be almost twice the height of the thinner parts.

A further structural detail that could be observed and resolved was a periodic height fluctuation along the contour of the fibrils. This was systematically observed for all fibrils with heights over $3 \mathrm{~nm}$. The presence of periodic fluctuations in height along the contour length of amyloid fibrils is controversial and a matter of debate in the literature. Gosal and colleagues, who studied fine filaments, were unable to observe any periodic structure in $\beta$-lactoglobulin fibrils ${ }^{15,17}$. In the same systems, however, Arnaudov and colleagues ${ }^{19}$ observed a periodic structure with a period of $\sim 26 \mathrm{~nm}$. When compared to previous studies on $\beta$-lactoglobulin amyloid fibrils, our results show, for the first time, the presence of a periodic structure with different periods. Figure 3 presents AFM images of fibrils with four different periods (35, 75, 100 and $135 \mathrm{~nm}$ ), corresponding to fibril maximum heights of $4,6,8$ and $10 \mathrm{~nm}$, respectively. Thus, the periodicity is found to increase linearly with the height of the fibrils.

'Laboratoire de Physique de la Matière Vivante, Ecole Polytechnique Fédérale de Lausanne (EPFL), CH-1015 Lausanne, Switzerland, ${ }^{2}$ Department of Physics and Fribourg Center for Nanomaterials, University of Fribourg, Ch. Musée 3, CH-1700, Fribourg, Switzerland, ${ }^{3}$ Laboratoire de Biophysique Statistique, Ecole Polytechnique Fédérale de Lausanne (EPFL), CH-1015 Lausanne, Switzerland, ${ }^{4}$ ETH Zurich, Food \& Soft Materials Science, Institute of Food, Nutrition \& Health, LFO23, Schmelzbergstrasse 9, 8092 Zürich, Switzerland. *e-mail: giovanni.dietler@epfl.ch; raffaele.mezzenga@agrl.ethz.ch 

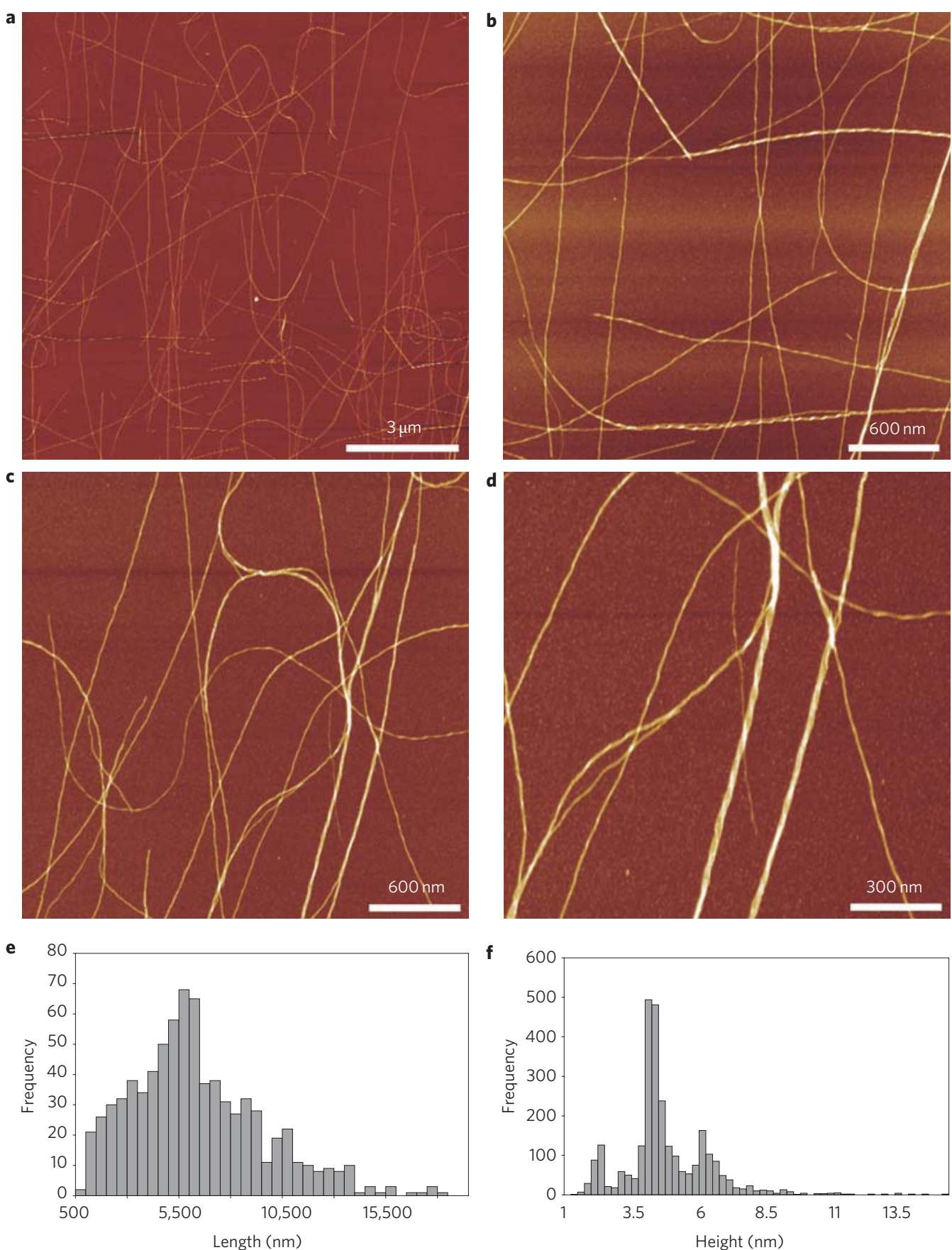

Figure 1 | AFM imaging, contour length and maximum height distribution of $\boldsymbol{\beta}$-lactoglobulin fibrils. a-d, Tapping-mode AFM height images of $\beta$ lactoglobulin amyloid fibrils at different magnifications. e, Distribution of contour length of $\beta$-lactoglobulin fibrils ranging from $0.5 \mu \mathrm{m}$ to $15 \mu \mathrm{m}$, with a maximum at $5.5 \mu \mathrm{m}$. $\mathbf{f}$, Histogram of $\beta$-lactoglobulin fibril maximum heights with three major peaks corresponding to maximum measured heights of $\sim 2,4$ and $6 \mathrm{~nm}$.

The combination of fibril height distribution, fibril periodicity and fibril splitting/thinning can be explained by a hierarchical multistranded self-assembly scheme. These elements, taken together, strongly suggest that the $\beta$-lactoglobulin fibrils have a helical lefthanded structure. Similarities between the formation of $\beta$-lactoglobulin fibrils and amyloid protein aggregation have so far only been suggested, but not confirmed ${ }^{17,19}$. Amyloid fibrils obtained by different aggregation mechanisms and from other proteins do show evidence of helical structures formed by twisting of single filaments around each other ${ }^{26,27}$. Our AFM studies of $\beta$-lactoglobulin fibrils not only demonstrate analogies between heat-induced protein fibrils and amyloid fibrils, but also clearly reveal similarities in their self-assembly structure and mechanisms, suggesting a possible general model for amyloid fibril assembly.

To further reinforce our self-assembly picture of the aggregation of single filaments into different classes of multistranded helical fibrils, we analysed the evolution of persistence length with fibril height. The persistence length $l_{\mathrm{p}}$ is determined directly from the AFM images by means of the bond correlation function for semiflexible polymers in a two-dimensional conformation, $<\cos \theta(s)>\approx \exp \left(-s / 2 l_{\mathrm{p}}\right)$, where $\theta$ is the angle between the tangent vectors to the chain at two points separated by a contour distance $s$ (Fig. $4 \mathrm{a}$ ) and the factor 2 is used to rescale the exponential decay to account for the two-dimensional nature of protein 

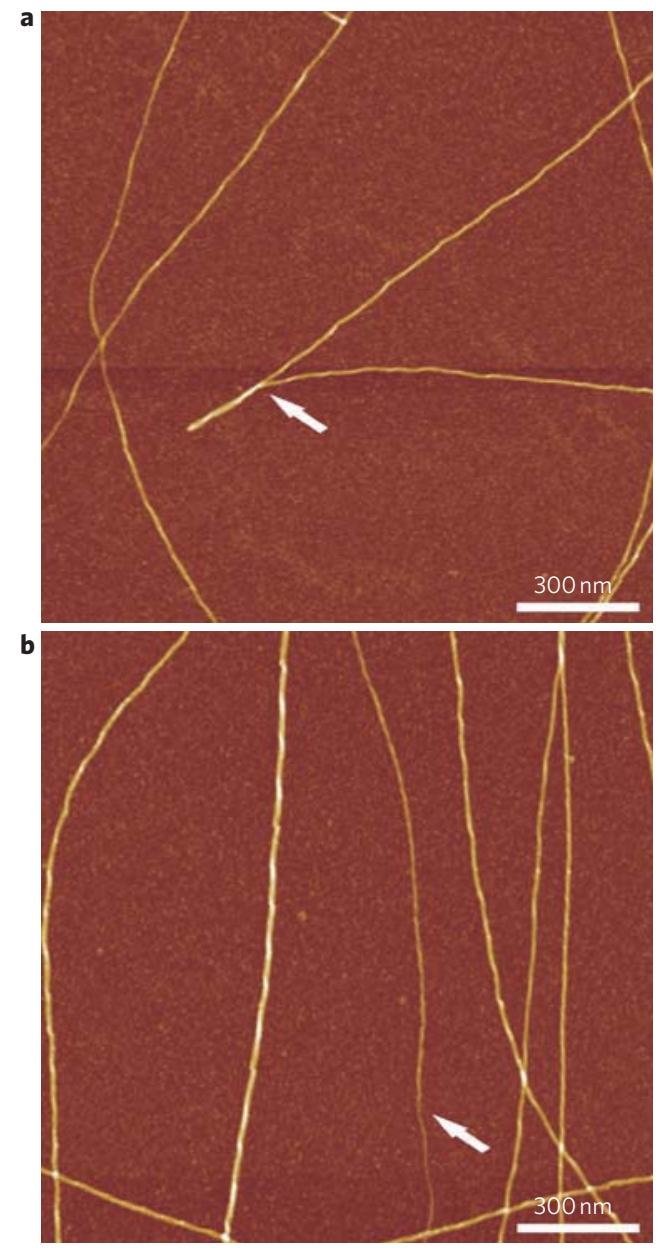

Figure 2 | AFM imaging of fibril splitting and thinning. a, AFM height image of fibril splitting, in which a single thick fibril unwinds into two separate thinner fibrils. The height of thicker, non-split fibril was found to be almost double that of thinner, split parts. b, AFM image of fibril thinning. In this case the thicker fibril tends to be thinner at its end. Again, the ratio of the height far from the fibril end and at the chain end was found to be approximately two.

fibrils absorbed on a substrate ${ }^{28}$. The persistence length $l_{\mathrm{p}}$ is the length above which thermal fluctuations can buckle a rod-like polymer, and it is therefore a measure of the elastic properties of a molecule ${ }^{29} . l_{\mathrm{p}}$ is given by the relation $l_{\mathrm{p}}=E I / k_{\mathrm{B}} T$, where $E$ is the Young's modulus of the polymer, $I$ the area moment of inertia of the polymer cross-section, $k_{\mathrm{B}}$ the Boltzmann constant and $T$ the temperature.

In Fig. $4 \mathrm{~b}$, the correlation function $<\cos \theta(s)>$ between tangent vectors at a contour distance $s$ is given for the heights of the three main populations of fibrils. Fitting of data in Fig. $4 \mathrm{~b}$ with the exponential decay given above directly yields the values of the persistence length for the three main populations of fibrils, and these are plotted in Fig. $4 \mathrm{c}$ against the respective maximum heights. This statistical treatment clearly indicates a linear increase of the persistence length with fibril cross-section. A simple scaling argument can be used to further assess the multistranded helical self-assembly scheme of the protein fibrils investigated.

For a multistranded fibril, the area moment of inertia $I$ depends directly on the packing geometry when packing individual filaments together. Essentially two packing mechanisms can be envisaged for a multistranded helical fibril: a close-packing filaments model and a ribbon-like packing scheme $e^{27,30,31}$.

When assuming that individual filaments are close-packed together, the multistranded fibril cross-section can be approximated
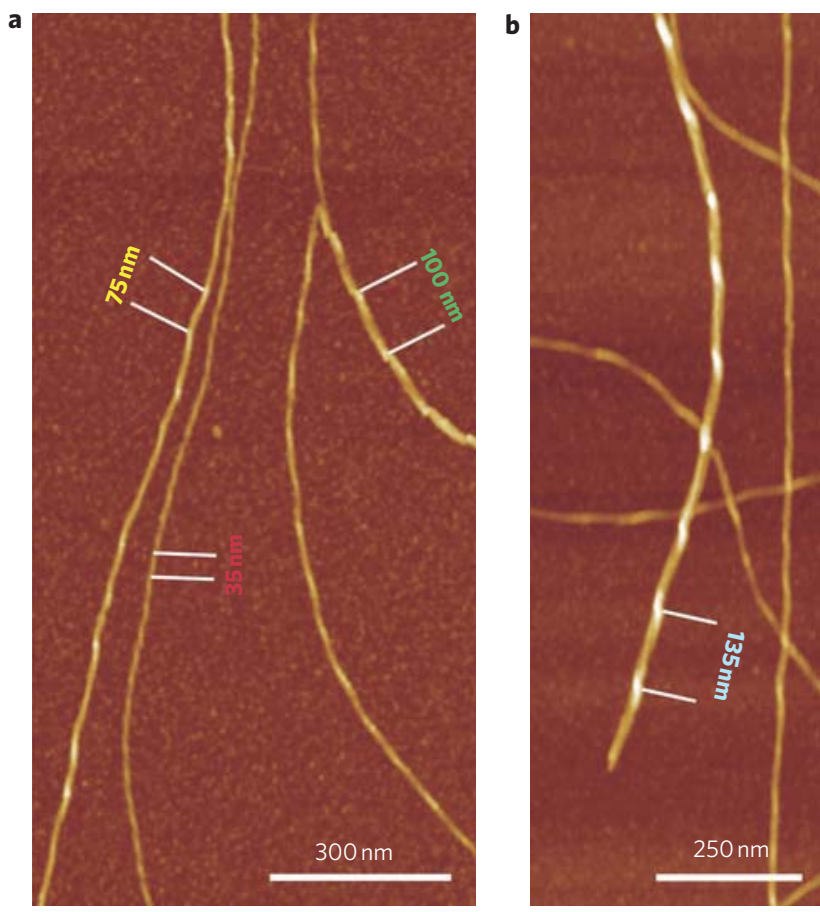

Figure 3 | Different periods of $\boldsymbol{\beta}$-lactoglobulin fibrils. a, AFM height image of fibrils with a period of $35 \mathrm{~nm}$ (for fibrils with a maximum height of $4 \mathrm{~nm}$ ) $75 \mathrm{~nm}$ (maximum height, $6 \mathrm{~nm}$ ) and $100 \mathrm{~nm}$ (maximum height, $8 \mathrm{~nm}$ ). b, Example of a fibril with a period of $135 \mathrm{~nm}$ and a maximum height of $10 \mathrm{~nm}$.

by a cylinder of radius $r$. In the presence of a multitude of $n$ filaments of area $A_{0}$, the total cross-sectional area would then be $A \approx n A_{0} \approx r^{2}$, which leads to an increase in the cross-section (and thus maximum fibril height) with the number of filaments of the form $r \approx n^{1 / 2}$. The corresponding area moment of inertia $I$ scales as $r^{4}$ and thus quadratically with the cross-sectional area $A: I \approx$ $r^{4} \approx A^{2} \approx n^{2}$, leading to a scaling behaviour of the persistence length with number of filaments of the form $l_{\mathrm{p}} \approx n^{2} E A_{0}^{2} / k_{\mathrm{B}} T$.

Neither the growth of the cross-section nor the increase in persistence length expected for close-packed filaments is compatible with the experimental behaviour observed in Fig. If and 4c, respectively, so a close-packed mechanism for forming filaments into a multistranded helical fibril can be ruled out.

For a multistranded fibril formed by a twisting ribbon of $n$ filaments, the cross-section can be approximated by a rectangle of height $2 r_{0}$ and width $2 n r_{0}$, where $r_{0}$ is the radius of the single filament. In this case, the observable radius of the fibril, and thus the maximum observable height, are simply proportional to the number of filaments $\left(r \approx n r_{0}\right)$. Furthermore, because the geometry is no longer axisymmetric, two area moments of inertia exist (corresponding to the bending modes with respect to the two main symmetry planes of the ribbon), scaling respectively as $I \approx r_{0} \cdot\left(n r_{0}\right)^{3}$ and $I \approx\left(r_{0}\right)^{3} \cdot n r_{0}$. Because the persistence length is proportional to the energy (in units of $k_{\mathrm{B}} T$ ) needed to bend the fibril, it essentially reflects the lowest energy bending mode, that is, the one with the lowest area moment of inertia $I \approx\left(r_{0}\right)^{3} \cdot n r_{0}$, leading to a scaling behaviour of the persistence length of the form $l_{\mathrm{p}} \approx n r_{0}^{4} E / k_{\mathrm{B}} T$. Thus, for a multistranded helical fibril with twisting ribbon-like cross-section, both the scaling behaviour of the maximum height and the persistence length have a linear relationship with the number of filaments in the fibril, in perfect agreement with the results in Fig. If and 4c. Further experimental evidence of the ribbon-like structure is provided by the fluctuations in height found along the fibrils. Figure $4 \mathrm{~d}$ shows those measured for fibrils with a maximum height of $6 \mathrm{~nm}$ : the most frequent 
a
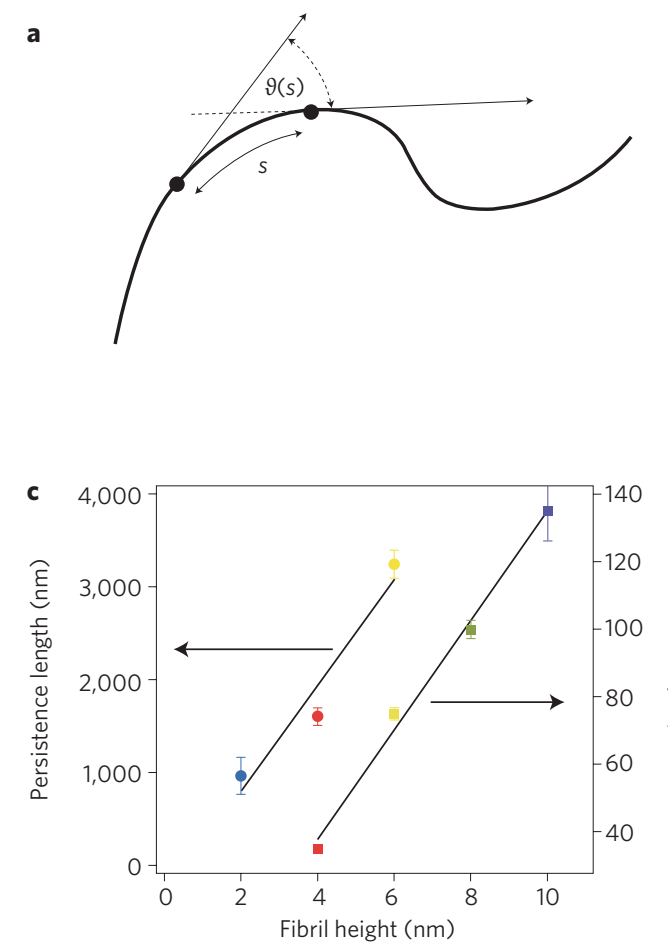

b
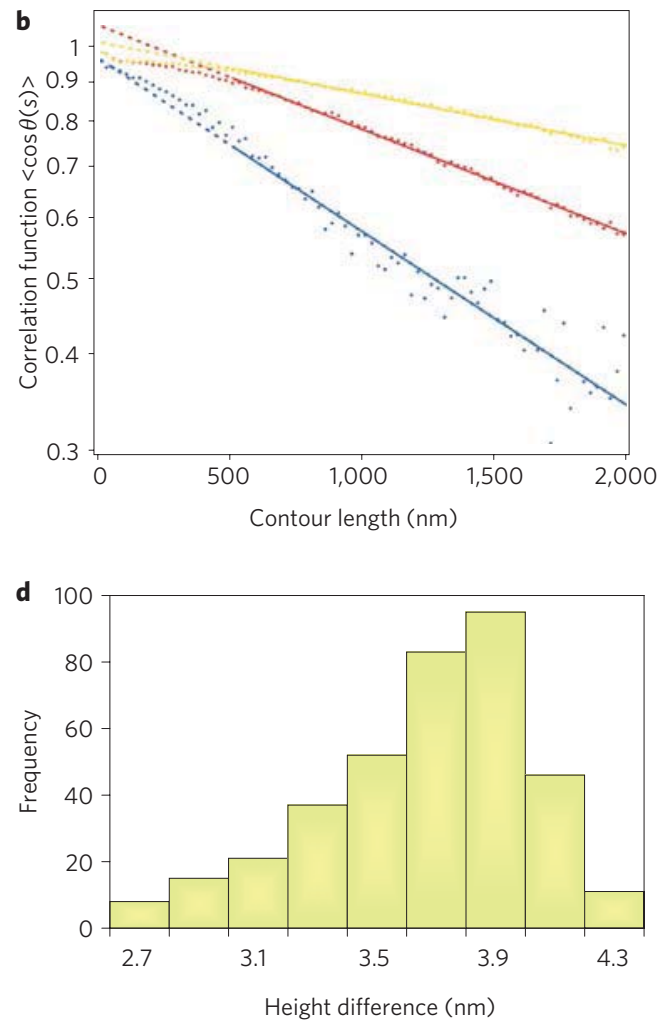

e

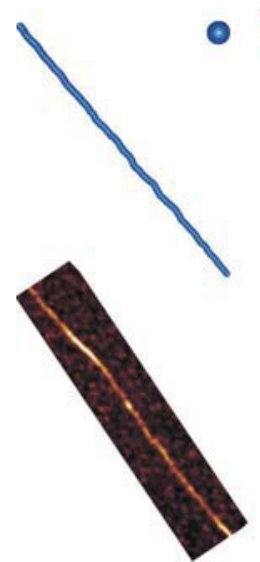

๑

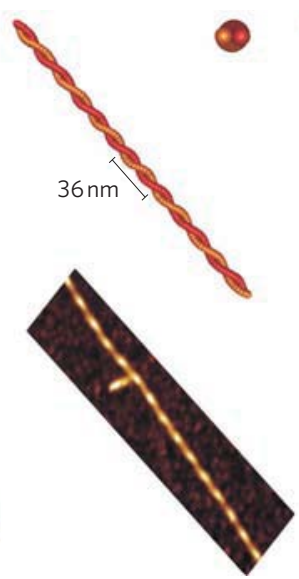

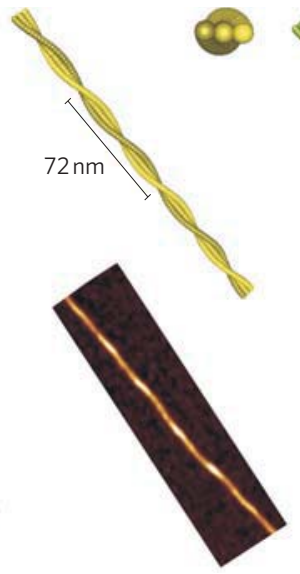
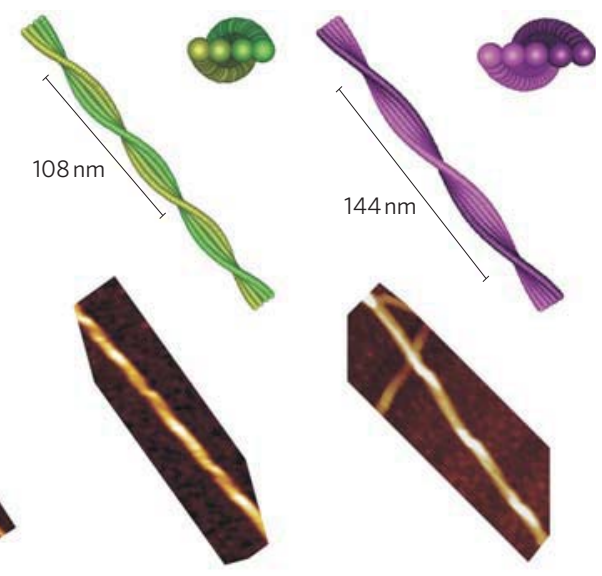

Figure 4 | Linear relation between the number of filaments and persistence length and periodicity of $\boldsymbol{\beta}$-lactoglobulin fibrils. a, Sketch of a semiflexible fibril chain with contour distance $s$ and angle $\theta(s)$ among rigid fibril segments, used to determine the bond correlation function $\langle\cos \theta(s)\rangle$. $\mathbf{b}$, Decay of $<\cos \theta(s)>$ as a function of contour length $s$ for filaments with heights of 1.5-2.5 nm (blue points, 50 fibrils)), for fibrils with heights of 3.5-4.5 nm (red points, 108 fibrils) and $5.5-6.4 \mathrm{~nm}$ (yellow points, 60 fibrils). For populations with heights over $6 \mathrm{~nm}$, counts were too few to allow a meaningful statistical analysis of the persistence length (compare with Fig. 1f). Fitting with a single exponential decay yields $l_{\mathrm{p}}=968 \mathrm{~nm}$ (blue), 1,620 nm (red) and 3,244 nm (yellow). The fits start at $s=500 \mathrm{~nm}$, because for lower $s$ values the correlation function is distorted by the finite resolution of the AFM images.

c, Persistence length and pitch of fibrils plotted against fibril cross-section for the populations of fibrils with maximum heights of $2 \mathrm{~nm}$ (blue), $4 \mathrm{~nm}$ (red), $6 \mathrm{~nm}$ (yellow), $8 \mathrm{~nm}$ (green) and $10 \mathrm{~nm}$ (violet), respectively. $\mathrm{d}$, Distribution of the height difference between the maximum and minimum height along fibrils with a maximum height of $6 \mathrm{~nm}$. The fluctuation occurring most frequently $(4 \mathrm{~nm})$ corresponds to the difference in heights between a vertically and horizontally absorbed ribbon. Consistently, for fibrils with maximum heights of 8 and $10 \mathrm{~nm}$ (not shown), the maximum difference in height is found to be respectively $6 \mathrm{~nm}$ and $8 \mathrm{~nm}$, corresponding again to the difference between vertically and horizontally absorbed ribbons in four and five strands. e, AFM images and corresponding coarse-grain molecular dynamics reconstructions of left-handed helical fibril formation from the twisting of multistranded ribbons, with the number of filaments ranging between 1 and 5.

height fluctuation found at $3.9 \mathrm{~nm}$ corresponds to the height difference between a vertically absorbed ribbon (height $6 \mathrm{~nm}$ ) and a horizontally absorbed ribbon (height $2 \mathrm{~nm}$ ). Identical conclusions can be drawn for fibrils with maximum heights of 8 and $10 \mathrm{~nm}$.
Finally, the linear relation between the number of filaments in a fibril and its period (Fig. 4c) can also be rationalized by considering the balance between the different protein-protein interactions along the axis of the fibril. Although filaments can overcome electrostatic repulsion to aggregate into a common ribbon, it can be argued that 
repulsion is responsible for the twisting of the ribbon so as to minimize the total energy of the system. Indeed, two protein layers arranged consecutively along the axis of the fibril do repel each other, and the conformation that would minimize such repulsion would be, if rotations were completely free, with the two layers oriented at $90^{\circ}$ with respect to one another. However, consecutive layers are not free to rotate, because of the strong interactions that keep fibrils together. If consecutive layers are tilted with respect to one another by a small angle $\theta$, proteins in the outermost filament are sheared with respect to each other at a distance $d=(n-1)(b / 2) \theta$, where $n$ is the number of filaments and $b$ is the distance between the axes of adjacent filaments in a fibril. To keep filaments intact, $d$ must be smaller than a certain threshold $d_{\max }$, beyond which filaments would break. For a strong enough repulsion we assume that $d \approx d_{\max }$, which in turn implies $\theta \approx 2\left(d_{\max } / b\right) /(n-1)$ (obviously defined for at least two filaments). The period of the fibril corresponds to a number $m$ of layers such that $\theta m=2 \pi$, and thus $m \approx \pi\left(b / d_{\max }\right)(n-1)$. The period is then found to be proportional to $n-1$, again in perfect agreement with experimental observations (Fig. 4c).

Figure $4 \mathrm{e}$ outlines the hierarchical helical structure emerging from the present study for amyloid-like fibrils. The schematic representations from single-filament up to five-stranded twistingribbon helices as reconstructed by coarse-grain molecular dynamics simulations (see Supplementary Information) are given, together with the corresponding AFM images. Different projections of the multistranded helical fibril reconstructions are also available in separate movies in the Supplementary Information.

In summary, by performing a statistical analysis on single-molecule AFM images, we were able to demonstrate analogies between heat-denaturated $\beta$-lactoglobulin fibrils and amyloidal fibrils, inferring a general amyloidosis process in both classes of materials. Cross-section, persistence length and height periodic fluctuations along the contour length of the fibrils allowed us to establish that single-filament, double-, triple- and multistranded left-handed helical aggregates all occur under equilibrium conditions, and that the most frequently occurring population appears to be the double-stranded helix. These results were tested against simple scaling arguments, which also supported the picture emerging from AFM analysis, and allowed us to determine that the helical periodicity in these fibrils originates from the twisting of a ribbon-like cross-section.

These results shine a new light on the structure of amyloidal protein fibrils. In particular, they show that a strong tendency to inter-fibril aggregation exists, even for fibrils such as those based on heat-denaturated $\beta$-lactoglobulin at $\mathrm{pH} 2$, for which a positive linear charge density as high as $+20 e$ per monomer is present ${ }^{32}$. This suggests that strong repulsive electrostatic forces and low ionic strength are not sufficient alone to prevent amyloidal aggregation, and that a strong energetic driving force must exist to promote aggregation under these conditions. Once aggregation has occurred, the electrostatic repulsive forces induce a twisting of the observed ribbon. Possible reasons for the attractive forces leading to aggregation might arise from the amphotheric nature of the protein fibrils, in which neutral residues occurring periodically along the contour length of the fibrils can promote strong attractive 'hydrophobic' interactions ${ }^{32}$. The occurrence of a ribbon-like cross-section rather than the more compact close-packing of single filaments may very well arise from the balance of hydrophobic short-range attraction and electrostatic long-range repulsion.

\section{Methods}

BioPURE- $\beta$-lactoglobulin was obtained from Davisco Foods International. The powder was dialysed and freeze-dried before use, as described in literature ${ }^{16}$ A $2 \mathrm{wt} \%$ solution was prepared by dissolving the powder in Milli-Q water at room temperature over at least $1 \mathrm{~h}$. The solution was centrifuged at $10,800 \mathrm{~g}$ over a period of $1 \mathrm{~h}$ at $20^{\circ} \mathrm{C}$ using a Sorvall RC3C Plus centrifuge (DuPont), adjusted to $\mathrm{pH} 2$, and filtered through a $0.45-\mu \mathrm{m}$ Millipore filter before heat treatment. Finally, the solution was distributed in 100-ml flasks, which were hermetically sealed and placed in a water bath for heating at $90{ }^{\circ} \mathrm{C}$ for $5 \mathrm{~h}$. Each flask contained a magnetic bar to stir the solution during the heating process. After heat treatment, the flasks were immediately cooled by immersion in ice-water mixtures to quench the aggregation process. Solutions of $0.1 \mathrm{wt} \% \beta$-lactoglobulin fibrils were prepared by dilution using $\mathrm{pH} 2$ Milli-Q water. The final $\mathrm{pH}$ was checked once again and adjusted if needed.

A $10-\mu \mathrm{l}$ aliquot of $0.1 \mathrm{wt} \%$ solution was deposited onto freshly cleaved mica, incubated for $2 \mathrm{~min}$, rinsed with Milli-Q water and dried by nitrogen. Images used for the statistical analysis of the fibrils were acquired on dried samples to allow the highest possible resolution, but complementary experiments in a wet environment ( $\mathrm{pH}$ 2) were also carried out to rule out potential artifacts in the preparation method, confirming the validity of sample preparation. Images were collected using a Nanoscope IIIa (Veeco) operated in tapping mode in air. Images were simply flattened using the Nanoscope III software, and no further image processing was carried out. The contour length of fibrils was measured with Ellipse $\mathrm{e}^{33}$.

Received 29 October 2009; accepted 1 March 2010; published online 11 April 2010

\section{References}

1. Caughey, B. \& Lansbury, P. T. Protofibrils, pores, fibrils and neurodegeneration: separating the responsible protein aggregates from the innocent bystanders. Annu. Rev. Neurosci. 26, 267-298 (2003).

2. Stradner, A. et al. Equilibrium cluster formation in concentrated protein solutions and colloids. Nature 432, 492-495 (2004).

3. Mezzenga, R., Schurtenberger, P., Burbidge, A. \& Michel, M. Understanding foods as soft materials. Nature Mater. 4, 729-740 (2005).

4. Selkoe, D. J. Folding proteins in fatal ways. Nature 426, 900-904 (2003).

5. Chiti, F. \& Dobson, C. M. Amyloid formation by globular proteins under native conditions. Nature Chem. Biol. 5, 15-22 (2009).

6. Knowles, T. P. et al. Role of intermolecular forces in defining material properties of protein nanofibrils. Science 318, 1900-1903 (2007).

7. Nelson, R. et al. Structure of the cross-beta spine of amyloid-like fibrils. Nature 435, 773-778 (2005)

8. Chiti, F. \& Dobson, C. M. Protein misfolding, functional amyloid and human disease. Annu. Rev. Biochem. 75, 333-366 (2006).

9. Dobson, C. M. Protein folding and misfolding. Nature 426, 884-890 (2003).

10. Graumann, P. L. Cytoskeletal elements in bacteria. Annu. Rev. Microbiol. 61, 589-618 (2007).

11. Kueh, H. Y. \& Mitchison, T. J. Structural plasticity in actin and tubulin polymer dynamics. Science 325, 960-963 (2009).

12. Pearce, F. G., Mackintosh, S. H. \& Gerrard, J. A. Formation of amyloid-like fibrils by ovalbumin and related proteins under conditions relevant to food processing. J. Agric. Food Chem. 55, 318-322 (2007).

13. Kavanagh, G. M., Clark, A. H. \& Ross-Murphy, S. B. Heat-induced gelation of globular proteins: part 3. Molecular studies on low $\mathrm{pH}$ beta-lactoglobulin gels. Int. J. Biol. Macromol. 28, 41-50 (2000).

14. Bolder, S. G., Sagis, L. M., Venema, P. \& van der Linden, E. Effect of stirring and seeding on whey protein fibril formation. J. Agric. Food Chem. 55, 5661-5669 (2007).

15. Gosal, W. S., Clark, A. H. \& Ross-Murphy, S. B. Fibrillar beta-lactoglobulin gels: Part 1. Fibril formation and structure. Biomacromolecules 5, 2408-2419 (2004).

16. Jung, J. M., Savin, G., Pouzot, M., Schmitt, C. \& Mezzenga, R. Structure of heatinduced beta-lactoglobulin aggregates and their complexes with sodium-dodecyl sulfate. Biomacromolecules 9, 2477-2486 (2008).

17. Gosal, W. S., Clark, A. H., Pudney, P. D. A. \& Ross-Murphy, S. B. Novel amyloid fibrillar networks derived from a globular protein: beta-lactoglobulin. Langmuir 18, 7174-7181 (2002).

18. Veerman, C., Ruis, H., Sagis, L. M. \& van der Linden, E. Effect of electrostatic interactions on the percolation concentration of fibrillar beta-lactoglobulin gels. Biomacromolecules 3, 869-873 (2002).

19. Arnaudov, L. N., de Vries, R., Ippel, H. \& van Mierlo, C. P. Multiple steps during the formation of beta-lactoglobulin fibrils. Biomacromolecules 4, 1614-1622 (2003).

20. Bromley, E. H., Krebs, M. R. \& Donald, A. M. Aggregation across the lengthscales in beta-lactoglobulin. Faraday Discuss. 128, 13-27 (2005).

21. Sagis, L. M., Veerman, C. \& van der Linden, E. Mesoscopic properties of semiflexible amyloid fibrils. Langmuir 20, 924-927 (2004).

22. Arnaudov, L. N. \& de Vries, R. Strong impact of ionic strength on the kinetics of fibrilar aggregation of bovine beta-lactoglobulin. Biomacromolecules 7 , 3490-3498 (2006).

23. Nilsson, M. R. Techniques to study amyloid fibril formation in vitro. Methods 34, 151-160 (2004).

24. Lashuel, H. A. \& Wall, J. S. Molecular electron microscopy approaches to elucidating the mechanisms of protein fibrillogenesis. Methods Mol. Biol. 299, 81-101 (2005). 
25. Ikeda, S. \& Morris, V. J. Fine-stranded and particulate aggregates of heatdenatured whey proteins visualized by atomic force microscopy. Biomacromolecules 3, 382-389 (2002).

26. Chamberlain, A. K. et al. Ultrastructural organization of amyloid fibrils by atomic force microscopy. Biophys. J. 79, 3282-3293 (2000).

27. Khurana, R. et al. A general model for amyloid fibril assembly based on morphological studies using atomic force microscopy. Biophys. J. 85, 1135-1144 (2003).

28. Witz, G., Rechendorff, K., Adamcik, J. \& Dietler, G. Conformation of circular DNA in two dimensions. Phys. Rev. Lett. 101, 148103 (2008).

29. Manning, G. S. Correlation of polymer persistence length with Euler buckling. Phys. Rev. A 34, 4467-4468 (1986).

30. Aggeli, A. et al. Hierarchical self-assembly of chiral rod-like molecules as a mode for peptide beta-sheet tapes, ribbons, fibrils and fibers. Proc. Natl Acad. Sci. USA 98, 11857-11862 (2001).

31. Paravastu, A. K., Leapman, R. D., Yau, W. M. \& Tycko, R. Molecular structural basis for polymorphism in Alzheimer's beta-amyloid fibrils. Proc. Natl Acad. Sci. USA 105, 18349-18354 (2008).

32. Jung, J. M. \& Mezzenga, R. Liquid crystalline phase behavior of protein fibers in water: experiments versus theory. Langmuir 26, 504-514 (2010).
33. Marek, J. et al. Interactive measurement and characterization of DNA molecules by analysis of AFM images. Cytometry A 63, 87-93 (2005).

\section{Acknowledgements}

The authors thank G. Witz and J. Vieira for helpful discussions and assistance during the experiments.

\section{Author contributions}

J.A. performed AFM imaging, analysed data and wrote the paper. J.M.J. prepared the fibrils. J.F. ran coarse-grain molecular dynamics simulations of the fibrils and analysed data P.D.L.R and G.D. analysed data and wrote the paper. R.M designed the study, analysed the data and wrote the paper. All authors discussed the results and commented on the manuscript.

\section{Additional information}

The authors declare no competing financial interests. Supplementary information accompanies this paper at www.nature.com/naturenanotechnology. Reprints and permission information is available online at http://npg.nature.com/reprintsandpermissions/. Correspondence and requests for materials should be addressed to G.D. and R.M. 